\section{PTU-021 LONG-TERM PALLIATIVE ABDOMINAL DRAINS VS LARGE-VOLUME PARACENTESIS IN CIRRHOSIS-RELATED REFRACTORY ASCITES: MULTI-CENTRE FEASIBILITY RCT (REDUCE)}

\begin{abstract}
1,2Lucia Macken*, ${ }^{2}$ Louise Mason, ${ }^{3}$ Heather Gage, ${ }^{3}$ Jake Jordan, ${ }^{3}$ Morro Touray,

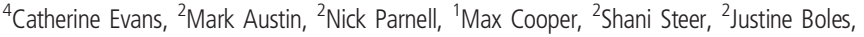
${ }^{1}$ Stephen Bremner, 'Debbie Lambert, 'David Crook, ${ }^{2}$ Gemma Earl, ${ }^{2}$ Jean Timeyin, ${ }^{5}$ Sam Thomson, ${ }^{7}$ David Sheridan, ${ }^{8}$ Peter Isaacs, ${ }^{6}$ Mark Wright, ${ }^{1,2}$ Ahmed Hashim, 1,2Sumita Verma. 'Brighton And Sussex Medical School, Brighton, UK; ${ }^{2}$ Brighton and Sussex University Hospitals NHS Trust, Brighton, UK; ${ }^{3}$ University of Surrey, UK; ${ }^{4}$ Kings College London, London, UK; ${ }^{5}$ Western Sussex Hospitals NHS Foundation Trust, Worthing, UK; ${ }^{6}$ University Hospital Southampton NHS Foundation Trust, Southampton, UK; ${ }^{7}$ University Hospitals Plymouth NHS Trust, Derriford, UK; ${ }^{8}$ Blackpool Teaching Hospitals NHS Foundation Trust, Blackpool, UK
\end{abstract}

\subsection{6/gutjnl-2019-BSGAbstracts.230}

Introduction Ascites develops in about 90\% with advanced cirrhosis; when refractory to medical therapy, standard of care is repeated large volume paracentesis (LVP) with albumin support. Refractory ascites (RA) confers a median life expectancy of six months without liver transplantation (LT). LVP is not an optimal palliative strategy. One alternative is long-term abdominal drains (LTAD), used in advanced malignant ascites, also enabling community management. Our ultimate aim is to improve end of life care (EoLC) in advanced cirrhosis and RA. This feasibility randomised controlled trial (RCT) aimed to resolve uncertainties in designing a definitive RCT.

Methods Multicentre feasibility RCT with 1:1 randomisation between standard of care (LVP) vs. LTAD (Rocket Medical) in adults with RA, ineligible for LT. Both arms received prophylactic antibiotics. LTAD were inserted under ultrasound guidance. Community nurses undertook home visits to drain ascites dependent on symptoms; (maximum 6L/week), without albumin support. Follow up was 12 weeks with home visits every two weeks for the following assessments: clinical, questionnaire based to include quality of life, palliative care needs, carer burden and health economics (HE). Here we report clinical and HE outcomes.

Results Thirty six patients were randomised; 19 LVP (two withdrew, wanting LTAD) and 17 LTAD (one withdrew insufficient ascites). Mean age (years) LTAD vs. LVP $66 \pm$ 10.4 vs. $68 \pm 12$; predominately male ( $76 \%$ vs. $74 \%)$. Participants were well matched at baseline in liver tests and prognostic scores: LTAD vs. LVP (serum bilirubin ( $\mu \mathrm{mol} / \mathrm{L}) 26 \pm$ 15.8 vs. $16 \pm 10$, serum albumin (g/L) $33 \pm 4.2$ vs. $31 \pm$ 3.3 , serum creatinine $(\mathrm{mmol} / \mathrm{L}) 113 \pm 46.7$ vs.118 \pm 53.1; MELD $14 \pm 4.6$ vs. $16 \pm 7.2$ ). One LTAD participant required hospitalisation for repeated LVP. Serum albumin $(\mathrm{g} / \mathrm{L})$ in the LTAD arm declined to $29 \pm 3.3$ at week two, subsequently remaining stable LTAD vs. LVP $(29 \pm 5.6$ vs. $31 \pm$ 5.5). Serum creatinine remained stable in both arms. There were no LTAD related serious adverse reactions. LTAD related adverse reactions included mild cellulitis $(n=4)$ and small volume leakage around LTAD insertion site $(n=3)$, all resolving rapidly. Peritonitis was rare, LTAD (possible) $\mathrm{n}=1$ and LVP $\mathrm{n}=2$. Overall mortality was $36 \%$ (12/33). Mortality and median survival (days in those who died) were $7 / 16$ (44\%) vs. 5/17 (29\%), 53 days (IQR 43) vs. 61 days (IQR 35) in LTAD vs. LVP respectively. All but one death was liver related. Those in LTAD arm spent $\approx 20 \%$ less time in hospital. All nine alive in the LTAD arm at end of study elected to keep LTAD in. Detailed clinical and HE analysis is underway.

Conclusions Preliminary data from the REDUCe study supports the safety and efficacy of palliative LTAD in RA due to advanced cirrhosis. LTAD allows successful management in the community with reduction in health resource utilisation. Proceeding to a definitive RCT is justified.

\section{PTU-022 ABNORMAL LIVER FUNCTION TESTS: CURRENT TIME TO REFERRAL AND USING ILFTS}

${ }^{1}$ lain Macpherson*, ${ }^{2}$ Matthew Priest, ${ }^{3}$ Ellie Dow, ${ }^{3}$ John Dillon, ${ }^{2}$ Andrew Fraser. ${ }^{1} N H S$ Forth Valley, Larbert, UK; ${ }^{2}$ Queen Elizabeth University Hospital, Glasgow, UK; ${ }^{3}$ Ninewells Hospital, Dundee, UK

\subsection{6/gutjnl-2019-BSGAbstracts.231}

Introduction At present, patients with abnormal LFTs referred to secondary care undergo a serological liver screen and Fibroscan at a nurse-led pre-assessment clinic, before being discussed at a multi-disciplinary team meeting (MDT). The outcome is either advice for management in Primary Care or they are offered an appointment at a consultant clinic. 'Intelligent Liver Function Tests' (iLFTs) provide a diagnostic pathway and stratification for patients with abnormal LFTs in primary care. When requesting iLFTs, the referrer is asked to include alcohol intake, BMI, and if there are features of the metabolic syndrome. Patients with abnormal LFTs on screen undergo reflex testing without further venepuncture or review. We aimed to assess how long it took from initial abnormal liver enzymes to referral to secondary care and compared the outcomes to what would have happened if iLFTs were the initial test.

Methods A retrospective analysis of 93 cases of abnormal LFTsbrought to MDT from February-August 2018 was performed. The MDT result was compared with the diagnosis and outcome had iLFTsbeen used. In addition, the number of times patients had abnormal LFTs and the time period in which the patient had been known to have abnormal LFTs was recorded.

Results 37 patients were referred back to primary care from the MDT. $23(24.7 \%)$ would not have been referred if iLFTs were used.

50 patients were offered review at a consultant clinic. Only $2(2.1 \%)$ patients would not have been referred if iLFTs had been used; both had low fibrosis risks. In $41 / 50$ cases (82\%), the diagnosis at MDT was the same if the GP had used iLFTs.

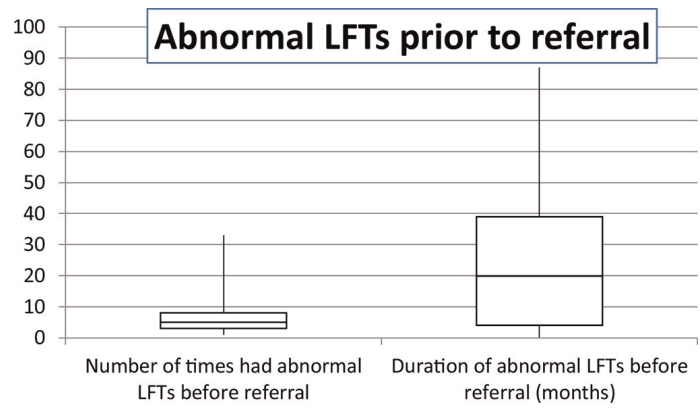

Abstract PTU022 Figure 1 Abnormal LFTs prior to referral: the number of times a patient had abnormal LFTs and the length of time they were known to have LFTs before referral to clinic 